\title{
A Comparative Analysis of Tools for Verification of Security Protocols
}

\author{
Nitish Dalal, Jenny Shah, Khushboo Hisaria, Devesh Jinwala \\ Department of Computer Engineering, \\ S.V. National Institute of Technology, Ichchhanath, Surat, India \\ E-mail:dcj@svnit.ac.in \\ Received July 20, 2010; revised August 21, 2010; accepted September 24, 2010
}

\begin{abstract}
The area of formal verification of protocols has gained substantial importance in the recent years. The research results and subsequent applications have amply demonstrated that the formal verification tools have indeed helped correct the protocols even after being standardized. However, the standard protocol verification tools and techniques do not verify the security properties of a cryptographic protocol. This has resulted in the emergence of the security protocol verifiers to fill the need. In this paper, taking the two popular security verification tools namely Scyther and ProVerif as the basis, we identify a few security protocols and implement them in both Scyther and ProVerif, to aptly evaluate the tools, in terms of the security properties of the selected protocols. In the process, we not only characteristically present a comparative evaluation of the two tools, but also reveal interesting security properties of the protocols selected, showing their strengths and weaknesses. To the best of our knowledge, this is a unique attempt to juxtapose and evaluate the two verification tools using the selected security protocols.
\end{abstract}

Keywords: Formal Verification, Security Protocols, Attacks

\section{Introduction}

A protocol is a set of rules that followed the defined conventions to establish semantically correct communications between the participating entities. A security protocol is an ordinary communication protocol in which the message exchanged is often encrypted using the defined cryptographic mechanisms. The mechanisms Symmetric Key Cryptography or Asymmetric Key Cryptography are used to obtain various cryptographic attributes such as Confidentiality, Entity Authentication, Message Integrity, Non-repudiation, Message Freshness, to name a few [1]. However, merely using cryptographic mechanisms, does not guarantee security-wise semantically secure operation of the protocol, even if it is correct. There indeed have been reported breaches in the security protocols, after being published and accepted as a safe protocol [2-4]. In such a scenario, in case of the ordinary communication protocols, recourse has been taken to the rigorous verification of the same using appropriate tool for the domain. As for example, the protocol verifier SPIN is used to verify the communication protocols for distributed software [5].
Such successful use of the formal methods for verification has led to the upsurge in devising similar tools for verifying the security properties of a cryptographic protocol, too. In order to gain confidence in the cryptographic protocol employed, it has been found desirable that the protocol be subjected to an exhaustive analysis that verifies its security properties. Some of the tools developed for the purpose are Scyther [6], ProVerif [7], Athena [8], Avispa [9], Casper/FDR to name a few. These tools differ in their input language and also in the way they verify the protocols and provide the output.

However, an important fall-out of the emergence of a plethora of such tools is that, it often becomes difficult for a security engineer to identify the appropriateness and suitability of a tool for the protocol under consideration. Motivated with this difficulty, we in this research report, document our attempt at evaluating the two popular cryptographic verification tools namely ProVerif and Scyther. We use six popular cryptographic protocols to implement the same and then analyze the protocols, using both these tools. In the process, interestingly, we not only comparatively evaluate the tools under consid- 
eration, but also identify various interesting properties of the protocols used.

To the best of our knowledge, this is a unique attempt to juxtapose and evaluate the two verification tools using the security protocols namely the Kao Chow Authentication Protocol [10], the 3-D Secure Protocol [11], the Needham Schroeder Public Key Protocol [3], the Andrew Secure RPC Protocol [12], the Challenge Handshake Authentication Protocol [13] and the DiffieHellman Key Exchange Protocol [14].

The rest of this paper is organized as follows: in section 2, we describe the theoretical background which includes a brief introduction of Scyther and ProVerif tools. In section 3, we formally define the problem and survey the related work. In section 4 , we present details of our implementation of various protocols, whereas do a comparative analysis of the two tools, before we draw the conclusion and show probable future work, in the last section.

\section{Theoretical Background}

\subsection{Cryptographic Verification Tools}

As mentioned before, one can find a series of tools for the verification of the cryptographic protocols. We have selected Scyther and ProVerif amongst all these, for the comparative evaluation. This decision is largely driven by the popularity of these two tools amongst all, we surveyed. In this section we depict the vital characteristics of these two tools.

\subsubsection{Scyther}

Scyther is a tool used for security protocol verification, where it is assumed that all the cryptographic functions are perfect. The tool provides a graphical user interface that makes it easier to verify and understand a protocol. In addition, attack graphs are generated whenever an attack is found corresponding to the claim mentioned. The tool can also verify all the possible claims on the protocol. The tool can be used to find problems that arise from the way the protocol is constructed. It can also be used to generate all the possible trace patterns. The verification here can be done using a bounded or an unbounded number of sessions. The language used to write protocols in Scyther is SPDL (Security Protocol Description Language) [6].

\subsubsection{ProVerif}

ProVerif is a software tool for automated reasoning about the security properties found in cryptographic protocols. This was developed by Bruno Blanchet. This tool verifies the protocol for an unbounded number of sessions, using unbounded message space. The tool is capable of attack reconstruction-wherein if a property cannot be proved, an execution trace which falsifies the desired property is constructed. There are two ways of providing input to this tool-Horn clauses or Pi calculus. In both cases, the output of the tool is essentially the same. Explicit modeling of attacker is not required. It is also possible to state whether an attacker is active or passive [7].

\section{Related Work}

The main objective of our research is to dissect the two protocol verification tools, Scyther and ProVerif, and to provide a comparative analysis of the two. In order to analyze the tools, suitable standard input protocols are required to be identified. After careful observation of a series of such protocols, we have identified, implemented and analyzed six different security protocols using both these tools. As mentioned earlier, these protocols are namely the Kao Chow Authentication Protocol, the 3-D Secure Protocol, the Needham Schroeder Public Key Protocol, the Andrew Secure RPC Protocol, the Challenge Handshake Authentication Protocol and the Diffie Hellman Key Exchange Protocol.

One can find a few attempts in the literature that concentrate on tools used for protocol verification, whereas very few of them provide a comparative analysis of protocol verification tools as in [15] and in [16]. However, there is no attempt that either focuses on or subsumes a detailed comparative analysis of the tools Scyther and Proverif, using the actual implementation of protocols as the basis. Hence, we believe our attempt here to be a unique one of its kind.

In the next section, we discuss briefly the implementation of each of the protocol in Scyther as well as in ProVerif and analyze the same.

\section{Implementation and Analysis}

\subsection{Kao Chow Authentication Protocol}

\subsubsection{Definition}

The Kao Chow protocol is a mutual authentication and key distribution protocol aiming at strong authentication and low message overhead. A trusted third party, S, is used to generate and distribute keys. It is the responsibility of $\mathrm{S}$ to generate a fresh secret session key $\mathrm{k}$. The two communicating parties, $\mathrm{A}$ and $\mathrm{B}$, will use this key to encrypt future message exchanges. Both the parties have 
secret keys shared with the server, Kas and Kbs, which are used to encrypt and decrypt any messages that are exchanged. Further, the protocol aims to authenticate the parties A and B to each other using their nonces $\mathrm{Na}$ and $\mathrm{Nb}$ [10]. The pseudocode of a typical protocol execution run is shown in Figure 1.

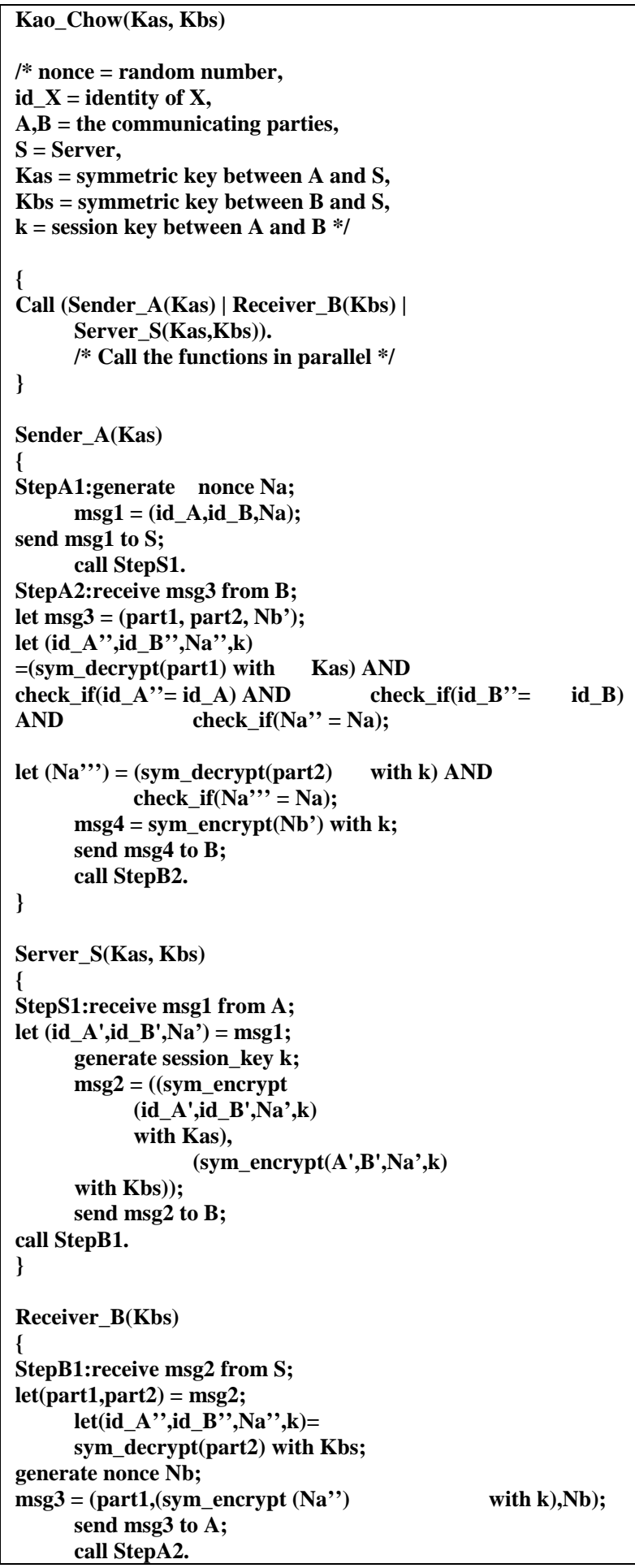

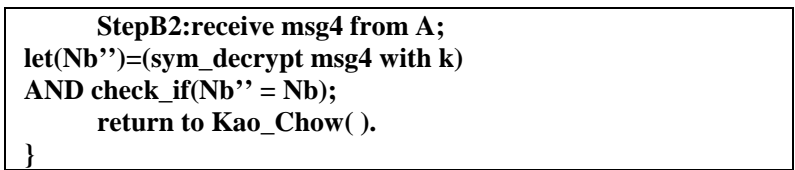

Figure 1. Kao Chow authentication protocol.

\subsubsection{Analysis}

When this protocol is verified using Scyther, attacks are found on the sender side as well as on the receiver side. However, we observe that the session key on the sender side (A) is secret whereas it is compromised on the receiver side. Because of this, there are synchronization and agreement attacks on both the sides. For the claim to check the secrecy of session key on the receiver (B) side, scyther outputs saying the claim is "Falsified" and that there is "At least 1 attack." When a similar query is written for the initiator side, scyther gives the output that the claim has been "Verified" and that there are "No attacks".

When the same protocol is analyzed using ProVerif, we obtain a similar result. That is, it can also detect that the session key is not secret on the receiver side. The private free variable var is encrypted using the session key $\mathrm{k}$ and published over a public channel $c$, the output obtained is false i.e., the key is not secret on the initiator side. When a similar query is provided for the receiver side, ProVerif gives the output saying that session key is secret on the receiver side. When the parameter "attacker" is set to passive, we do not obtain any attacks showing that there are no passive attacks on the protocol. The symmetric key Kas is secret on $A$ side as well as the server(S) side. $K b s$ is also secret on the server side as well as B side.

\subsection{3-D Secure Protocol}

\subsubsection{Definition}

The 3-D Secure is an XML-based protocol used as an added layer of security for online credit and debit card transactions. Developed by Visa, its aim is to improve the security of Internet payments. It is offered to customers as the Verified by Visa service. It has also been adopted by MasterCard, under the name MasterCard SecureCode.

A transaction using Verified by Visa/SecureCode will initiate a redirect to the website of the card issuing bank to authorize the transaction. Each Issuer could use any kind of authentication method. The most common approach is a password-based method. Thus, to effectively buy on the Internet means using a secret password tied to the card. The Verified by Visa protocol recommends the bank's verification page to load in an inline frame session. In this way, the bank's systems can be held responsible for most security leaks [11]. 
The pseudocode of the protocol is shown in Figure 2.

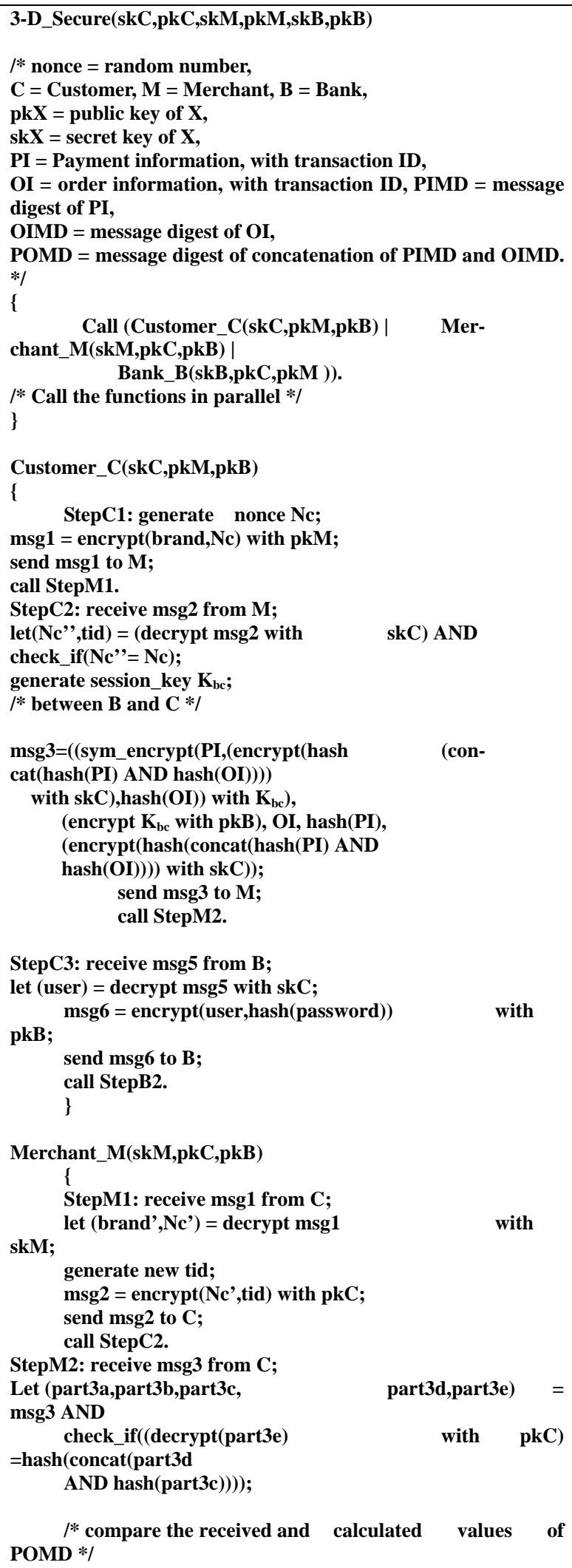

StepC3: receive msg5 from $B$;

let (user) = decrypt msg5 with skC;

pkB; msg6 = encrypt(user,hash(password)) with

send msg6 to $B$;

call StepB2.

\}

Merchant_M(skM,pkC,pkB)

\{

StepM1: receive msg1 from C;

let (brand',Nc') = decrypt msg1

skM;

generate new tid;

msg2 = encrypt(Nc',tid) with pkC;

send msg2 to $C$;

call StepC2.

StepM2: receive msg3 from $\mathrm{C}$;

Let (part3a,part3b,part3c,

msg3 AND

check_if((decrypt(part3e)

$=$ hash(concat(part3d

AND hash(part3c))));

POMD */

(con-

generate session_key $\mathbf{K}_{\mathrm{bm}}$;

/* between $\mathrm{B}$ and $\mathrm{M} *$ /

msg4 = (part3a,part3b,(sym_encrypt (encrypt tid with skM) with $\quad K_{b m}$ ),(encrypt $K_{b m}$ with pkB));

send msg4 to $B$;

call StepB1.

StepM3: receive msg7 from $B$;

Let (part7a,part7b,part7c,part7d) =

let $\left(\mathrm{K}_{\mathrm{mb}}\right)=$ decrypt (part7b) with skM;

let $\left(\right.$ tid' $\left.^{\prime}\right)=\left(\operatorname{decrypt}\left(\mathbf{s y m} \_\right.\right.$decrypt $\quad$ part7a with $\left.\mathbf{K}_{\mathrm{mb}}^{\prime}\right)$ with pkB) AND check_if(tid'=tid);

msg8 = (part7c,part7d, $($ encrypt

crypt (tid,amount)

with skM) with pkB)));

send msg8 to $B$;

call StepB3.

StepM4: receive msg9 from $B$;

let $($ tid') $=$ (decrypt msg9 with

skM)

AND check_if(tid' =tid);

return to 3-D_Secure( ).

Bank_B(skB,pkC,pkM):

\{

StepB1: receive msg4 from $M$;

let (part4a,part4b,part4c,part4d $)=$

msg4;

let $K_{b c}=$ decrypt (part4b) with skB;

let (PI,encrypted_POMD,OIMD) =

sym_decrypt(part4a) with $K_{b c}$;

let POMD = (decrypt(encrypted_POMD) with pkC)

AND check_if(POMD =

AND

OIMD)));

hash(concat(hash(PI)

POMD */

/* compare the received and calculated values of

let $\mathrm{K}_{\mathrm{bm}}=$ decrypt (part4d) with skB;

let tid = (decrypt(sym_decrypt

(part4c) with ks2) with pkM);

msg5 = encrypt user with pkC;

send msg5 to C; call StepC3.

StepB2: receive msg6 from C;

let (user',password') $=($ decrypt

skB) AND

check_if(user' = user);

generate session_key $K_{\text {'mb; }}$;

/* between $\mathrm{M}$ and $\mathrm{B}$ */

generate nonce $\mathrm{Nb}$;

msg7 = ((sym_encrypt (encrypt tid with skB $)$

with $\mathbf{K}_{\mathrm{mb}}^{\prime}$ ),

(encrypt K' ${ }_{m b}$ with pkM),

(sym_encrypt(encrypt Nb with skB) with $\mathrm{K}_{\mathrm{mb}}{ }_{\mathrm{mb}}$ ),

(encrypt $\mathrm{K}_{\mathrm{mb}}$ with pkB));

send msg7 to $M$;

call StepM3.

StepB3: receive msg8 from $M$;

let (part8a,part8b,part8c) = msg8;

let $\left(K^{\prime}{ }_{m b}\right)=$ decrypt $($ part8b) with

let $\left(\mathrm{Nb}^{\prime}\right)=($ decrypt $($ sym_decrypt

$\mathrm{K}_{\text {mb) }}$ ) with pkB) AND check_if(Nb' $\left.=\mathrm{Nb}\right)$; let $($ tid', ,amount $)=($ decrypt $\quad$ (de-

crypt(part8c) with skB)

with pkM) AND

check_if(tid' = tid); 
msg9 = encrypt tid with pkM;

send msg9 to $M$;

call StepM4.

$$
\text { \} }
$$

Figure 2. 3-D secure protocol.

\subsubsection{Analysis}

On analyzing this protocol with Scyther, we find that for five runs of the protocol, there are no attacks on the customer(C) and merchant(M) side. However, the attacks are found on the bank(B) side. The session key $K_{b c}$ and the customer's password are secret on $\mathrm{C}$ side. On the $\mathrm{M}$ side, we find that the session keys $K_{b m}, K^{\prime}{ }_{m b}$ and $K_{b c}$ are secret. On analyzing the claims on B side, we find that the session key $K_{m b}^{\prime}$ and the customer's password are secret. But, the keys $K_{b c}$ and $K_{b m}$ can be compromised here. These attacks bring a limitation of the tool Scyther to the fore. In Scyther, we have no provision of comparing the values of two variables. For instance, in step 4, the bank receives POMD, PI and hash (OI). However, there is no way that we can write an "if" condition in this tool to check the equality of the received POMD and the calculated POMD (calculated using the function "hash" and the received value of hash(OI) and PI). The attacks that we see here are the result of such deficiencies in the tool. Had there been a way to compare values here, no attacks would have been found. In order to obtain attacks on session keys, a special "key-compromise" part needs to be added to the protocol. In this module, we first specify who the communicating parties are. Next, we provide the intruder with all the packets that have been used in a session and the values of the session keys for that session in order to verify if a freshness attack is possible.

When the 3D-secure protocol is analyzed using ProVerif, no attacks are found. That is, the tool says that this protocol is perfectly secure and that there is no way that an intruder can gain knowledge of either the session keys $\left(K_{b c}, K_{b m}, K_{m b}^{\prime}\right)$ or the customer's password. This is because, in this tool we have the provision of writing an "if" condition to check for the equality of two values. For instance, in step B1, we see that the value POMD obtained after decrypting a part of the message can be compared to the calculated value of POMD (using the received values PI and OIMD and the hash function) and the protocol proceeds only if these two values are found to be the same. This way, all the attacks are countered and the protocol becomes completely secure.

Thus, we see that ProVerif provides this advantage over Scyther. Not being able to compare values in Scyther leads to attacks being found whereas in ProVerif, as the values can be compared, these attacks are not found.

\subsection{Needham-Schroeder Public Key Protocol}

\subsubsection{Definition}

The Needham-Schroeder Public Key Protocol, based on public-key cryptography, is intended to provide mutual authentication between two parties communicating on a network. It is assumed here that the two parties know the public key of the other. Thus, encrypting the data is possible using the public key of the other party. Here, A and $\mathrm{B}$ represent the communicating parties [3].

The pseudocode of the protocol is shown in Figure 3.

\subsubsection{Analysis}

On verifying this protocol using Scyther, attacks are found. It is seen that all the attacks are on the $\mathrm{B}$ (receiver) side. Both the nonces, $\mathrm{Na}$ and $\mathrm{Nb}$, can be obtained by the attacker. In addition, there are synchronization and agreement attacks. Thus, the protocol is not secure. In addition, Scyther provides the facility of observing all possible trace patterns. For this protocol, a single trace pattern is obtained on the A (initiator) side as there is no intrusion possible here.

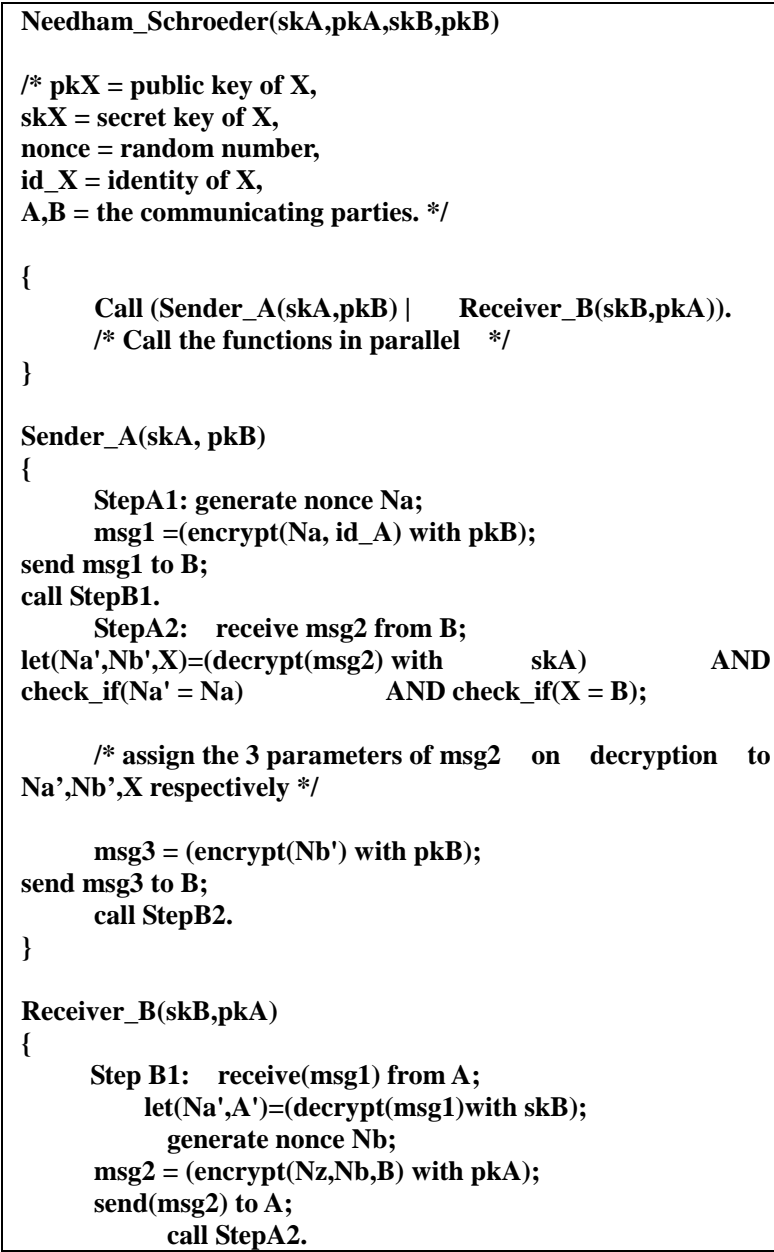




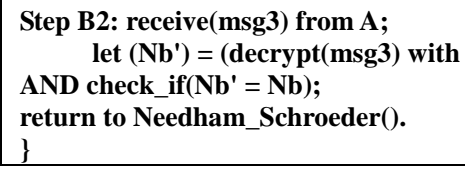

Figure 3. Needham schroeder public key protocol.

On B side, 2 trace patterns are obtained-one showing the normal run of the protocol and the other showing the man-in-the-middle attack. No attacks are found when the protocol is verified for a single run. But with 2 or greater runs, attacks are generated.

In ProVerif, the protocol is run for an unbounded number of times. For checking the secrecy of nonces and keys in ProVerif, a random number is generated and it is encrypted using the nonce and broadcasted over a public channel. The results obtained are similar to those obtained using Scyther. But, there is no way of checking for the synchronization and agreement attacks on either the receiver or the sender side.

\subsection{Andrew Secure RPC Protocol}

\subsubsection{Definition}

This protocol is intended to distribute a new session key between two parties A and B. The protocol must guarantee the secrecy of the new shared key $k$. In every session, the value of $k$ must be known only by the participants playing the roles of A and B. The protocol must guarantee the authenticity of $k$. In every session, on reception of message 4, A must be able to ensure that the key $k$ in the message has been created by A in the same session. The final message contains $N^{\prime} b$ which can be used in future messages as a handshake number [12].

The pseudocode of the protocol is shown in Figure 4.

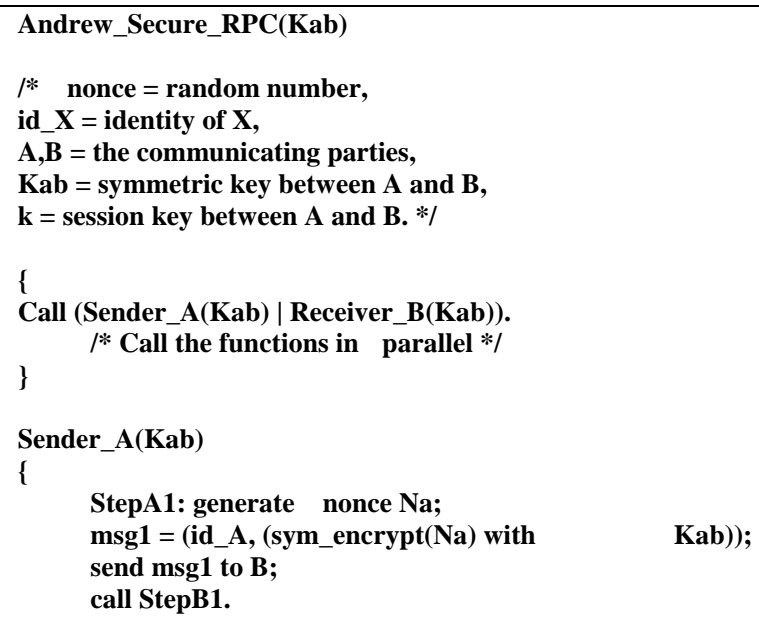

Kab));

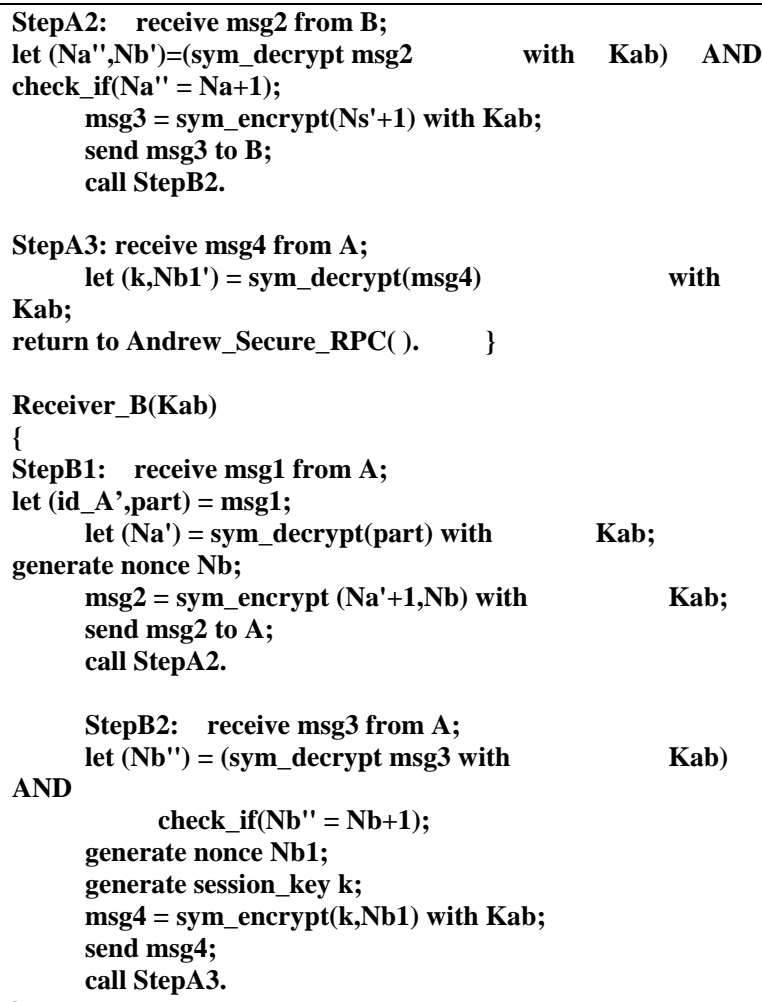

Figure 4. Andrew secure RPC protocol.

\subsubsection{Analysis}

When this protocol is verified using Scyther, attacks are found on the initiator side and none are obtained on the receiver side. The major attack is the one in which the session key is compromised. This is a freshness attack on the protocol. In this, an intruder can replay an old message (the last message) and the party A has no way of knowing that this has come from B or some intruder. Thus, the intruder can establish a session with A using an older session key. Since the communication is not taking place in the proper order, there are attacks of synchronization and agreement as well. For the claim to check the secrecy of session key $k$ on the initiator (A) side, scyther outputs saying the claim is "Falsified" and that there is "Exactly 1 attack". The attack graph provides a complete flow diagram of the actions of the parties and the intruder.

ProVerif also provides us with a similar result. That is, it can also detect that the session key is not secret on the initiator side. The private free variable var is encrypted using the session key $k$ and published over a public channel c, the output obtained is false i.e. the key is not secret on the initiator side. When a similar query is provided for the receiver side, ProVerif gives the output saying that session key is secret on the receiver side. In order to obtain a complete trace pattern, the parameter 
“traceDisplay" can be set to "long”. This provides an entire description of how the attack is executed. In addition, when the parameter "attacker" is set to "passive", we do not obtain any attacks showing that there are no passive attacks on the protocol. It can be verified that the symmetric key Kab is secret on both the sides.

\subsection{Challenge Handshake Authentication Protocol}

\subsubsection{Definition}

The Challenge Handshake Authentication Protocol (CHAP) uses a 3-way handshake to periodically verify the identity of the party. This is done upon initial link establishment, and may be repeated anytime after the link has been established. Once the link is established, the authenticator sends a challenge message to the party. The party responds with a value calculated using a one-way hash function. The authenticator checks the response against its own calculation of the expected hash value. If the values match, the authentication is acknowledged; otherwise the connection has to be terminated [13].

The pseudocode of the protocol is shown in Figure 5.

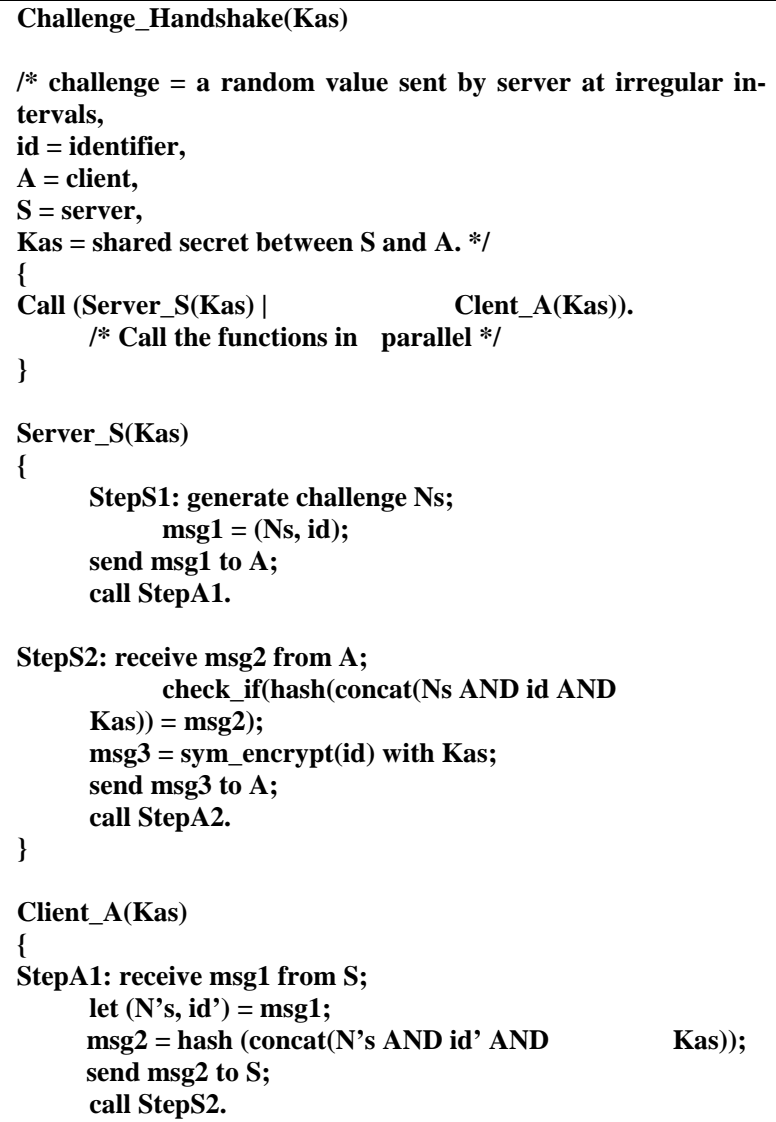

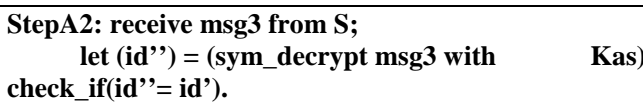

Figure 5. Challenge handshake authentication protocol.

\subsubsection{Analysis}

On analyzing this protocol with scyther, we see that the symmetric key (shared secret) is secret on both the sides, the server as well as the party which needs to be authenticated. But, there are synchronization and agreement attacks on A side but not on the server(S) side. These attacks are caused because the first message from the server is not encrypted. Thus, it can be captured by any intruder. But this does not lead to the shared secret being compromised as the message from the A party to the server is hashed using a one way hush function. Thus, even if the intruder knows the hash value and the hashing algorithm, there is no way to unhash the value and obtain the original message. A single trace pattern is obtained for the server side. For the A side, 2 patterns are obtained - one specifying the normal run of the protocol and the other giving details of how the communication can be disrupted.

When this protocol is verified using ProVerif, the output obtained shows that the symmetric key Kas cannot be compromised on either the client side or the server side. Thus, the communication is secure. In addition, when the parameter "attacker" is set to "passive", no attacks are found suggesting that there are no passive attacks on this protocol.

\subsection{Diffie-Hellman Key Exchange Protocol}

\subsubsection{Definition}

Diffie-Hellman key exchange (D-H) is a cryptographic protocol that allows two parties that have no prior knowledge of each other to jointly establish a shared secret key over an insecure communications channel. This key can then be used to encrypt subsequent communications using a symmetric key cipher. There are two publicly known numbers - a prime number $\mathrm{p}$ and a primitive root of that prime, g. Each party then chooses a random number which is less than $\mathrm{p}$ and using modular arithmetic, the key is calculated. Thus, a key is exchanged between two or more parties over an insecure channel [14].

The pseudocode of the protocol is shown in Figure 6.

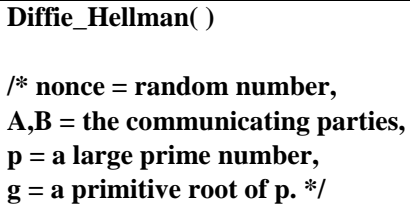




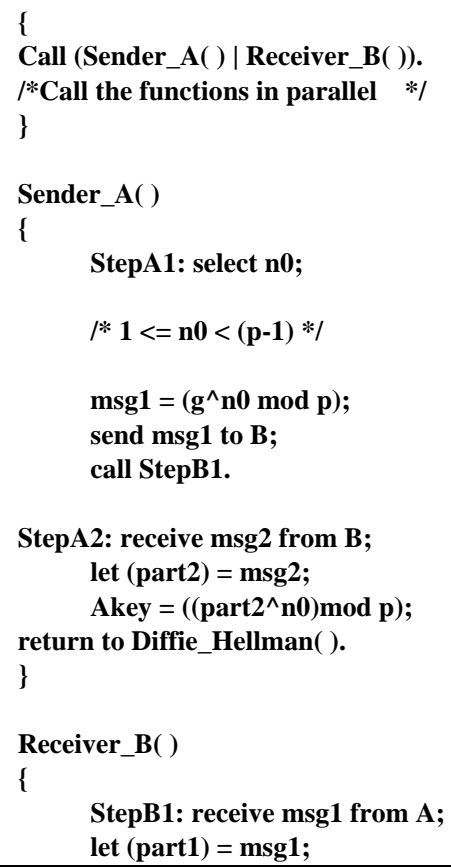

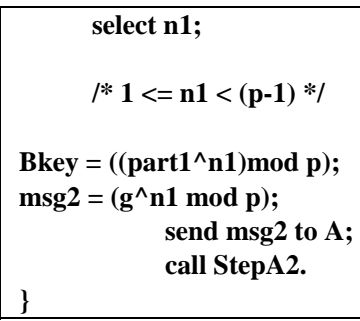

Figure 6. Diffie Hellman key exchange protocol.

\subsubsection{Analysis}

When this protocol is verified using ProVerif, we find that the generated key is not secret on the initiator side as well as the receiver side.

This is because of a man in the middle attack. An intruder is able to capture the public values from both the legitimate parties and send its own generated public value to both of them. Thus, an intruder is able to establish a session key with both the parties.

The Diffie Hellman key exchange cannot be modeled using Scyther. The reason is that there is no way to show

Table 1. Scyther and ProVerif characteristic comparison.

\begin{tabular}{|c|c|}
\hline Characteristics of SCYTHER & Characteristics of PROVERIF \\
\hline $\begin{array}{l}\text { - The protocol is modeled using the "spdl" language. } \\
\text { - It is possible to run the protocol for either bounded or } \\
\text { unbounded number of sessions. }\end{array}$ & $\begin{array}{l}\text { - The protocol is modeled using horn clauses or pi calcu- } \\
\text { lus. } \\
\text { - Tool has to be run through command line interface. }\end{array}$ \\
\hline $\begin{array}{l}\text { - } \quad \text { Tool comes with its own graphical user interface. } \\
\text { It generates the following possible outputs namely Prop- } \\
\text { erty holds for n runs, Property is false and attack trace is } \\
\text { shown, Property holds for all traces. }\end{array}$ & $\begin{array}{l}\text { - It generates the following possible outputs namely Prop- } \\
\text { erty is true, Property is false and attack trace is generated, } \\
\text { Property cannot be proven when false attack is found, } \\
\text { Tool might not terminate. }\end{array}$ \\
\hline $\begin{array}{l}\text { - Attack graphs are generated which give a visual flow } \\
\text { graph of a trace and are self explanatory. }\end{array}$ & $\begin{array}{l}\text { - } \quad \text { Step by step trace is generated explaining the run and } \\
\text { attack. }\end{array}$ \\
\hline $\begin{array}{l}\text { - All possible trace patterns are generated depicting proto- } \\
\text { col execution. }\end{array}$ & $\begin{array}{l}\text { - Trace is generated only for the property which is } \\
\text { checked. }\end{array}$ \\
\hline $\begin{array}{l}\text { - The communicating parties need to be modeled as roles. } \\
\text { - It doesn't provide any option to check for equality of } \\
\text { different variables. }\end{array}$ & $\begin{array}{l}\text { - The communicating parties need to be modeled as proc- } \\
\text { esses. } \\
\text { - Equality can be checked by using "if..then" or "let..in". }\end{array}$ \\
\hline $\begin{array}{l}\text { - Tool by its own discretion checks for secrecy of all possi- } \\
\text { ble variables, no explicit “claims” are necessary. }\end{array}$ & $\begin{array}{l}\text { - It checks only those attacks for which the "query" has } \\
\text { been specified in the code. }\end{array}$ \\
\hline $\begin{array}{l}\text { - The anticipated intruders along with the legitimate com- } \\
\text { municating parties have to be specified as agents. }\end{array}$ & - $\quad$ ProVerif does not require any such specification. \\
\hline $\begin{array}{l}\text { - In case of protocols which may suffer from a freshness } \\
\text { attack, we have to put a key compromise module in the }\end{array}$ & $\begin{array}{l}\text { - No special code for a freshness attack needs to be given } \\
\text { in ProVerif. }\end{array}$ \\
\hline code which specifies that a complete session has been & - $\quad$ Channels need to be specified for communication. \\
\hline captured and the intruder also knows the session key. & - It is possible to run the protocol only for an unbounded \\
\hline There is no concept of channels. & number of sessions. \\
\hline
\end{tabular}


the equivalence of 2 exponential operations. That is, a rule that states (exp (exp (g,x),y) mod p) and (exp (exp $(\mathrm{g}, \mathrm{y}), \mathrm{x}) \bmod \mathrm{p}$ ) are the same cannot be specified. Thus, it is not possible to handle such exponentiations which need equivalence conditions to be explicitly mentioned. Hence, the tool is not able to know that the keys that have to be generated on both sides are ideally the same.

\section{Conclusions and Future Work}

Based on the implementation and evaluation as described above, we summarize the comparative analysis of Scyther and ProVerif in the Table-I.

From this, it can be observed that applying formal methods to verify security protocols is an interesting and challenging research area. Using the tools Scyther and ProVerif, it is possible to model many security protocols in standard format, verify them and know the attacks they are susceptible to. We modeled six characteristic protocols and verified them using these tools. The tools vary in regards like their input language, manner in which the output is provided, the way in which traces of attacks are generated. Moreover, both the tools have a few limitations. Using these tools, it is easy to know what flaws these protocols suffer from so that the flaws can be rectified. Although verification using these tools does not ensure that the protocols once verified by these tools are flawless, still they provide a means to know many of the flaws easily. As a future work in this area, we plan to extend this comparative evaluation using other interesting protocols namely to have a better understanding of the differences in the cap- abilities of both. Our work can also be extended to model the same protocols using other tools to have a wider evaluation.

\section{Acknowledgements}

We are grateful to all those anonymous reviewers for their useful suggestions, to help give the paper the shape, it is now, in.

\section{References}

[1] W. Stallings, "Cryptography and Network Security: Principles and Practices," 4th Edition, Pearson Education, ISBN-10: 0131873164 ISBN-13: 9780131873162, 2006.

[2] D. Denning and G. Sacco, “Timestamps in Key Distribution Protocols," Communications of the ACM, Vol. 24, No. 8, 1981, pp. 533-536.
[3] R. Needham and M. Schroeder, "Using Encryption for Authentication in Large Networks of Computers," Communications of the ACMx, Vol.21, No. 12, 1978, pp. 993999.

[4] R. Needham and M. Schroeder, "Authentication Revisited,” ACM SIGOPS Operating Systems Review, Vol. 21, No. 1, 1987, p. 7.

[5] G. J. Holzmann, "Software Model Checking with SPIN," Advances in Computers, Vol. 65, 2005, pp. 78-109.

[6] C. J. F. Cremers, "Scyther-Semantics and Verification of Security Protocols,” Ph.D. Thesis, Eindhoven University of Technology, 2006.

[7] B. Blanchet, "An Efficient Cryptographic Protocol Verifier Based on Prolog Rules," Proceedings of the 14th IEEE Computer Security Foundations Workshop (CSFW), Cape Breton, IEEE Computer Society, 2009, pp. 82-96.

[8] D. Song, "Athena: A New Efficient Automatic Checker for Security Protocol Analysis," Proceedings of the12th IEEE Computer Security Foundations Workshop (CSFW), IEEE Computer Society, 1999, pp. 192-202.

[9] A. Armando et al., "The AVISPA Tool for the Automated Validation of Internet Security Protocols and Applications," Proceedings of Computer Aided Verification'05 (CAV), Vol. 3576 of Lecture Notes in Computer Science, Springer, 2005, pp. 281-285.

[10] I. Kao, Lung and R. Chow, "An Efficient and Secure Authentication Protocol Using Uncertified Keys," SIGOPS Operating Systems Review, Vol. 29, No. 3, 1995, pp. 14-21.

[11] Verified By Visa 3-D Secure Protocol. [Online] Available: https://usa.visa.com/personal/security/vbv/index.html, last retrieved on 2nd June 2010.

[12] M. Satyanarayanan, "Integrating Security in a Large Distributed System," ACM Transactions on Computer Systems, Vol. 7, No. 3, 1989, pp. 247-280.

[13] W. Simpson, "PPP Challenge Handshake Authentication Protocol (CHAP),” August 1996 http://www.ietf.org/rfc/ rfc1994.txt, last retrieved on 2nd June 2010.

[14] W. Diffie and M. E. Hellman, "New Directions in Cryptography," IEEE Transactions on Information Theory, Vol. 22, No. 6, 1976, pp. 644-654.

[15] C. J. Cremers, P. Lafourcade and P. Nadeau, "Comparing State Spaces in Automatic Security Protocol Analysis," Formal to Practical Security: Papers Issued from the 2005 -2008 French-Japanese Collaboration, Springer-Verlag, 2009, pp. 70-94.

[16] C. J. Cremers, "Unbounded Verification, Falsification, and Characterization of Security Protocols by Pattern Refinement," CCS'08: Proceedings of the 15th ACM conference on Computer and communications security, ACM Press, 2008, pp. 119-128. 